\title{
Effect of visual pattern on running an unpredictable maze
}

\author{
RICHARD D. WALK \\ George Washington University, Washington, D.C. 20052 \\ and \\ CLARENCE P. WALTERS \\ George Mason University, Fairfax, Virginia 22030
}

\begin{abstract}
Rats were trained to run through a 10-unit enclosed maze for a food reward. The maze pattern and the visual surface on the floor and walls of the maze varied from day to day. The most rapid speed of locomotion was for the stripes on the floors parallel to the route of the maze, and the all gray surface yielded the slowest running times. Stripes on the walls parallel with the route led to intermediate speeds. The results were relatively weak, but they have implications for studies of human locomotion.
\end{abstract}

The topic of this research is the visual stimulus for adequate locomotion. We used hooded rats as subjects as a basic approach to the problem.

An enclosed alley maze was constructed with a startbox and a goalbox. To make sure that the main difference was due to the visual surface, we varied both the configuration of the maze and the surface on it. By varying the configuration from day to day, we kept the rats from learning a maze pattern. Each animal was run through each type of visual surface and all 10 configurations. Illumination was kept at a low level; for humans, this would be at the approximate boundary of photopic and scotopic vision. Animals were pretrained to insure familiarity with the task before the testing itself began. The enclosed maze had no blind alleys. Latencies from startbox to goalbox were measured.

This research was carried out approximately concurrently with research with humans in a corridor maze in which speed of locomotion as affected by different stimulus patterns was among the variables investigated.

The alley segments had three possible types of visual surface: all gray, black and white stripes on the walls of the maze and a gray floor, and gray walls and black and white stripes on the floor. All stripes were parallel to the route of locomotion. Our hypothesis was that the black and white stripes on the floor would facilitate locomotion and that the all-gray maze would be slower. We made no hypothesis as to whether stripes on the walls would be similar to stripes on the floor or to the

This research was supported in part by NIH Biomedical Research Support Grant 2-S07-RR07019 and National Institute of Mental Health Grant MH25864 to George Washington University. Requests for reprints should be sent to Richard Walk, Department of Psychology, George Washington University, Washington, D.C. 20052. all gray, except we felt on intuitive grounds it would be somewhere in the middle.

\section{METHOD}

\section{Subjects}

The subjects were 12 hooded rats (Long-Evans) about 90 days old at the start of the experiment. The animals were food deprived to $80 \%$ of normal body weight and were maintained at that level throughout the experiment. Water was continuously available in the home cage.

\section{Apparatus}

The apparatus consisted of maze units that could be interchanged and rearranged to form different maze paths. Two basic maze units were used, one in the shape of a trapezoid and the other in the shape of a parallelogram. In addition, two startboxes and two goalboxes were required. These are illustrated, along with some possible maze routes, in Figure 1.

The elements were connected into a series of eight-unit mazes plus startbox and goalbox. The maze units had one of the three stimulus patterns: all gray, floors with black and white $1.3-\mathrm{cm}$ stripes parallel to the sides of the maze unit with gray walls, and gray floors with $1.3-\mathrm{cm}$ stripes on each wall, the stripes parallel to the long side. Each maze unit was covered with $1.3-\mathrm{cm}$ wire mesh.

The maze units were made of $1.9-\mathrm{cm}$ pine, and the inside dimensions of the maze were a width of $9.5 \mathrm{~cm}$ and a height of $12 \mathrm{~cm}$. The parallelogram units were $53 \mathrm{~cm}$ long, and the angle formed by the parallelogram was $45 \mathrm{deg}$. The trapezoidal units were $53 \mathrm{~cm}$ long through the center of the runway and thus had a $67-\mathrm{cm}$ base and a $41-\mathrm{cm}$ top (outside measurements). The angles were $45 \mathrm{deg}$ with the base and $135 \mathrm{deg}$ with the top. The startbox was $12.7 \mathrm{~cm}$ long on the inside and $9.5 \mathrm{~cm}$ wide and $12 \mathrm{~cm}$ high, but outside dimensions were $29 \mathrm{~cm}$ on one side and $15.2 \mathrm{~cm}$ on the other, leaving the same $45 \mathrm{deg}$ angle to join with the maze sections. A sliding Masonite door $10.5 \mathrm{~cm}$ wide and $15.2 \mathrm{~cm}$ high kept the animal in the startbox until it was released. The goalbox was like the startbox except for longer dimensions: The sliding door was $24 \mathrm{~cm}$ from the back. This produced outside dimensions of $41 \mathrm{~cm}$ on one side and $28 \mathrm{~cm}$ on the other. The two startboxes and two goalboxes were painted gray, with opposite long sides each a mirror image of the angle of the other. Each pattern was composed of eight maze units, four 



Figure 1. The top part of the figure shows the basic maze units required for the study. The bottom portion shows four possible maze paths $(S=$ start and $G=$ goal $)$.

parallelogram (two angled left, two angled right) and four trapezoidal ones. This allowed for flexibility in the construction of the eight-unit configurations. The total path for the animals was 8 times $53 \mathrm{~cm}$ or $4 \mathrm{~m} 24 \mathrm{~cm}$. Testing was conducted in dim light: External light was excluded, and three 15 -W bulbs reflecting light off the ceiling provided the only illumination.

\section{Procedure}

The animals were brought to the test room singly. Each subject was left in the startbox for $30 \mathrm{sec}$ prior to a test. Animals were tested at approximately the same time each day. Entrance into the goalbox was rewarded with two $45-\mathrm{mg}$ food pellets. Animals were fed enough at the end of a test session to maintain body weight. The experiment was divided into training and testing segments.

Training. The animals were given 10 days of training with two trials per day. They were first given 6 days of training, using the start- and goalboxes with a single maze unit between them. The maze units were randomly assigned so the animals had 2 days on each maze stimulus pattern. The last 4 days of pretraining, a six-unit maze was used and each stimulus pattern appeared twice in the six-unit maze. At the end of pretraining, the latencies from start- to goalbox were consistently below $20 \mathrm{sec}$.

Testing. The animals were shifted to the eight-unit maze for testing. A total of 10 different maze configurations were designed, and each stimulus pattern was used with every maze configuration. Animals had two trials per day and the stimulus pattern remained constant on a given day, but the maze configuration was changed between the first and second trials. Thus, 10 maze configurations times 3 stimulus patterns is 20 trials for each animal, or 15 days of testing. The maze units were cleaned after use and interchanged in their positions in the maze from day to day to control for possible cues. The 12 animals were run in two group of 6 animals each.

\section{RESULTS}

The individual data were somewhat hampered by variability. The median scores for each rat for each condition were computed for a Friedman's test for related samples to test for significance (Leach, 1979).

The medians of the median latencies from start to goal were as follows: stripes on floor, $11.1 \mathrm{sec}$; stripes on walls, $12.6 \mathrm{sec}$; gray, $13.3 \mathrm{sec}$. Using stripes on the floors as a base, the increment was $13 \%$ for stripes on walls and $20 \%$ for gray. This is somewhat of a difference, but not a large one.

The Friedman test rank orders each animal for each condition. The average rank for stripes on floor was 1.3 , for stripes on walls, 2.0 , and for gray, 2.7. The Friedman test itself revealed a $Q$ of $10.67(p<.01)$. Individual comparisons of the ranks for the conditions showed stripes on floors to be significantly faster than gray $(\mathrm{p}<.02)$, the only significant difference.

\section{DISCUSSION}

The experiment demonstrated that the visual surface can affect the running times for rats in an unpredictable maze. The main effect was confined to the visual pattern on the floor of the maze. The same pattern on the walls of the maze had a slight, but nonsignificant, effect on maze latencies. The surface around the feet, thus, appears to be the most important one for the rat and probably for many other species, including human beings.

Such a maze situation is of interest as an analogy to human locomotion through an uncertain visual environment. One thinks of individuals with visual defects as being particularly at risk in such an environment. A hint, not statistically significant, in our data may have some bearing on this issue. The increase in latencies compared with the base floor pattern for the allgray maze was $6 \%$ for the six rats with the fastest latencies on the floor pattern and $24 \%$ for the six rats with the slowest floor pattern latencies (the two-tailed level of significance is .10).

This pilot experiment was, in many respects, a disappointing one. The hypothesized effects were observed, but the effects were small, although one might maintain that a $20 \%-25 \%$ increase in speed has some practical importance. The possibility that different visual patterns may affect some subjects differently is a tantalizing one.

The main contribution of this study is the demonstration that different floor patterns affect speed of locomotion. The type of maze we used, with many different easily constructed paths, might also have applicability for other research.

\section{REFERENCE}

LEACH, C. Introduction to statistics. A nonparametric approach for the social sciences. Chichester, England: Wiley, 1979.

(Received for publication July 24,1981 .) 